\title{
Aligned vertical fractures, HTI reservoir symmetry, and Thomsen seismic anisotropy parameters for polar media
}

\author{
James G. Berryman ${ }^{1, *}$ \\ ${ }^{1}$ University of California, Lawrence Berkeley National Laboratory, \\ 1 Cyclotron Road, MS 90R1116, Berkeley, CA 94720, USA
}




\begin{abstract}
Sayers and Kachanov (1991) defined crack-influence parameters that are shown to be directly related to Thomsen (1986) weak-anisotropy seismic parameters for fractured reservoirs when the crack/fracture density is small enough. These results are then applied to the problem of seismic wave propagation in polar (i.e., non-isotropic) reservoirs having HTI seismic wave symmetry due to the presence of aligned vertical fractures and resulting in azimuthal seismic wave symmetry at the earth's surface. The approach presented suggests one method of inverting for fracture density from wave-speed data. It is also observed that the angular location $\theta_{e x}$ of the extreme value (peak or trough) of the quasi-SV-wave speed for VTI occurs at an angle determined approximately by the formula $\tan ^{2} \theta_{e x} \simeq \tan \theta_{m}=\left[\left(c_{33}-c_{44}\right) /\left(c_{11}-c_{44}\right)\right]^{1 / 2}$, where $\theta_{m}$ is an angle determined directly (as shown) from the $c_{i j}$ elastic stiffnesses, whenever these are known from either quasi-static or seismic wave measurements. Alternatively, $\theta_{e x}$ is given in terms of the Thomsen seismic anisotropy parameters by $\tan \theta_{e x} \simeq\left(\left[v_{p}^{2}(0)-v_{s}^{2}(0)\right] /\left[(1+2 \epsilon) v_{p}^{2}(0)-v_{s}^{2}(0)\right]\right)^{1 / 4}$, where $\epsilon=\left(c_{11}-c_{33}\right) / 2 c_{33}, v_{p}^{2}(0)=c_{33} / \rho$, and $v_{s}^{2}(0)=c_{44} / \rho$, with $\rho$ being the background inertial mass density. The axis of symmetry is always treated here as the $x_{3}$-axis for either VTI symmetry (due, for example, to horizontal cracks), or HTI symmetry (due to aligned vertical cracks). Then the meaning of the stiffnesses is derived from the fracture analysis in the same way for VTI and HTI media, but for HTI the wave speeds relative to the earth's surface are shifted by $90^{\circ}$ in the plane perpendicular to the aligned vertical fractures. Skempton's (1954) coefficient is used as a general means of quantifying the effects of fluids inside the fractures. Explicit formulas for Thomsen's parameters are also obtained for either drained or undrained fractures resulting in either VTI or HTI symmetry of the reservoir.
\end{abstract}

* JGBerryman@LBL . GOV 


\section{INTRODUCTION}

Aligned vertical fractures provide one commonly recognized source of azimuthal (surface angle dependent) seismic anisotropy in oil and gas reservoirs (Lynn et al., 1995). While layering in the earth also results in seismic anisotropy (Postma, 1955; Backus, 1962), horizontal layering of isotropic rock produces vertical transversely isotropic (VTI) media, and could not produce horizontal transversely isotropic (HTI) symmetry without some very significant uplift phenomena being present simultaneously. Of course, anisotropic layers such as shale beds (Hornby, 1994) bring seismic anisotropy with them too, but this anisotropy will more typically be VTI, rather than HTI.

While VTI earth media seem much easier to understand and analyze than HTI media, it is nevertheless true that techniques needed for analyzing HTI and VTI media are very similar. When the source of the anisotropy is aligned vertical fractures, we can make very good use of the simpler case of horizontal fracture analysis by introducing a rather minor change of our point of view that easily gives all the needed results. In particular, the use of Euler angle analysis (Goldstein, 1950) coupled with seismic wave reciprocity (Ben-Menahem and Singh, 1981) provides us with enough physical tools to avoid needing to do the mathematical rotations in our elastic equations.

Together with the simplifications already noted, we can understand very directly the sources of the anisotropy due to fractures by considering a method of analysis introduced by Sayers and Kachanov (1991), based on earlier work of Kachanov (1980). We find that elastic constants - and, therefore, the Thomsen (1986) anisotropy parameters - can be expressed very conveniently in terms of the Sayers and Kachanov (1991) formalism. Furthermore, in the very low crack density limit [which is also conceptually in line with the weak anisotropy approach of Thomsen (1986)], we can obtain direct links between the Thomsen parameters and the fracture properties. These links suggest a method of inverting for fracture density from seismic wave speed data. This approach is also known to be closely related to the formulation of the linear slip model of Schoenberg (1980), which has often been used in analyses of seismic waves in such fractured reservoirs (Hsu and Schoenberg, 1993; Bakulin et al., 2000).

The next section reviews Thomsen's weak anisotropy formulation (Thomsen, 1986). The third section introduces the crack-influence parameters (Sayers and Kachanov, 1991). The 
fourth and fifth sections discuss VTI and HTI symmetry - especially in the context of aligned horizontal and aligned vertical cracks - respectively. The sixth section shows one method of extending Thomsen's weak anisotropy formulation to larger angles, and, therefore, farther offsets. The seventh section discusses some details of the extreme values of the phase speed for quasi-SV-waves, and related issues for the quasi-P-waves. The final section discusses the results and summarizes our conclusions. Appendix A shows how to compute a certain angle $\theta_{m}$ directly from the Thomsen weak anisotropy parameters. Then, Appendix B summarizes some additional work on determining the value of $\theta_{e x}$, which is the angular location of the extreme point of the quasi-SV-wave speed in a VTI medium, based on the same information used in Appendix A. Finally, Appendix C makes use of Skempton's (1954) coefficient as a general means to quantify the effects of fluids inside the fractures. Explicit formulas for Thomsen's parameters are also obtained for either drained or undrained fractures resulting in either VTI or HTI symmetry of the reservoir.

\section{THOMSEN'S SEISMIC WEAK ANISOTROPY FORMULATION}

Thomsen's weak anisotropy formulation (Thomsen, 1986), having been designed specifically for use in short-offset velocity analysis in exploration geophysics, should be used only with great care for longer offsets. Approximations incorporated into the Thomsen formulas become most apparent for greater angles $\theta$ from the vertical, especially for quasicompressional and vertically polarized quasi-shear velocities $v_{p}(\theta)$ and $v_{s v}(\theta)$, respectively. The angle $\theta$ used as argument of these phase velocities is typically the one measured from the $\hat{z}$-vector pointing directly into the earth. For better results at larger offsets, one approach is to make use of the exact wave speeds, so we will need to discuss these well-known results as well.

For reference purposes, we include here the exact velocity formulas for quasi-P, quasi-SV, and SH seismic waves at all angles in a VTI elastic medium. These results are available in many places (Rüger, 2002; Musgrave, 2003), but were taken specifically from Berryman (1979) with some minor changes of notation (i.e., $a, b, c, f, l, m \rightarrow c_{11}, c_{12}, c_{33}, c_{13}, c_{44}, c_{66}$ ). The results for VTI media are well-known to be:

$$
v_{p}^{2}(\theta)=\frac{1}{2 \rho}\left\{c_{44}+\left[c_{11} \sin ^{2} \theta+c_{33} \cos ^{2} \theta\right]+R(\theta)\right\}
$$


and

$$
v_{s v}^{2}(\theta)=\frac{1}{2 \rho}\left\{c_{44}+\left[c_{11} \sin ^{2} \theta+c_{33} \cos ^{2} \theta\right]-R(\theta)\right\}
$$

where

$$
R(\theta)=\sqrt{\left[\left(c_{11}-c_{44}\right) \sin ^{2} \theta-\left(c_{33}-c_{44}\right) \cos ^{2} \theta\right]^{2}+4\left(c_{13}+c_{44}\right)^{2} \sin ^{2} \theta \cos ^{2} \theta}
$$

and, finally,

$$
v_{s h}^{2}(\theta)=\frac{1}{\rho}\left[c_{44}+\left(c_{66}-c_{44}\right) \sin ^{2} \theta\right] .
$$

While the $\mathrm{SH}$-wave is polarized as a true shear wave at all angles $\theta$, the quasi-P and quasi-SV waves both have mixed character at most angles. However, they do simplify to true P- and SV-waves at the $\theta$ 's for both the vertical and horizontal propagation directions.

Expressions for phase velocities in Thomsen's weak anisotropy limit can also be found many places, including Thomsen (1986, 2002), Rüger (2002), and Tsvankin (2005). The pertinent expressions for phase velocities in VTI media as a function of angle $\theta$, measured (as before) from the vertical direction, are

$$
\begin{gathered}
v_{p}(\theta) \simeq v_{p}(0)\left(1+\epsilon \sin ^{2} \theta-\frac{\epsilon-\delta}{4} \sin ^{2} 2 \theta\right), \\
v_{s v}(\theta) \simeq v_{s}(0)\left(1+\left[v_{p}^{2}(0) / v_{s}^{2}(0)\right] \frac{\epsilon-\delta}{4} \sin ^{2} 2 \theta\right),
\end{gathered}
$$

and

$$
v_{s h}(\theta) \simeq v_{s}(0)\left(1+\gamma \sin ^{2} \theta\right) .
$$

In our present context (i.e., for cracked/fractured reservoirs), the vertical phase velocities satisfy $v_{s}(0)=\sqrt{c_{44} / \rho_{0}}$, and $v_{p}(0)=\sqrt{c_{33} / \rho_{0}}$, where $c_{33}, c_{44}$, and $\rho_{0}$ are two stiffnesses of the cracked medium and the mass density of the isotropic host elastic medium. We explicitly assume that the cracks have insufficient volume to affect the mass density $\rho_{0}$ in a significant way. The three seismic parameters resulting from Thomsen's (1986) analysis for weak anisotropy with VTI symmetry are $\gamma=\left(c_{66}-c_{44}\right) / 2 c_{44}, \epsilon=\left(c_{11}-c_{33}\right) / 2 c_{33}$, and

$$
\delta=\frac{\left(c_{13}+c_{44}\right)^{2}-\left(c_{33}-c_{44}\right)^{2}}{2 c_{33}\left(c_{33}-c_{44}\right)}=\left(\frac{c_{13}+c_{33}}{2 c_{33}}\right)\left(\frac{c_{13}+2 c_{44}-c_{33}}{c_{33}-c_{44}}\right) .
$$

All three of these parameters can play important roles in the phase velocities given by (5)-(7) when the crack densities are high enough. If there are no other sources of anisotropy, and if crack densities are very low, then the quasi-SV shear wave will actually have no dependence 
on angle of wave propagation. This result follows from the fact that the so-called anellipticity parameter $A=\epsilon-\delta$, vanishes when $\epsilon \equiv \delta$, which we will soon see does happen in general at lower crack densities.

For each approximate phase speed, Thomsen's approximation has included a step that removes the square on the left-hand side of equations (1), (2), and (4) and expands a square

root of the right hand side. This Taylor series expansion introduces a factor of $\frac{1}{2}$ multiplying the $\sin ^{2} \theta$ terms on the right hand side, and - for example - immediately explains how equation (7) is obtained from (4). The other two equations for $v_{p}(\theta)$ and $v_{s v}(\theta)$, i.e., (5) \& (6), involve additional approximations as well and we will explain these in more detail as we progress through the analysis.

Figure 1 compares results obtained from the exact formulas (1)-(4), when applied to the case of aligned vertical fractures and HTI (horizontal transverse isotropy) symmetry. One pertinent observation arises for the $v_{s v}$ wave speeds in which the Figures 1(c) and 1(f) show very clearly that the peak (or in some other situations it might be a trough) for the exact model is usually not at $\theta_{e x}=45^{\circ}$, whereas Thomsen's approximation always places the peak or trough exactly at $45^{\circ}$. Understanding and resolving this discrepancy is one of the main purposes of the present paper. We develop a more flexible approximation that follows the correct behavior more closely.

\section{FRACTURED RESERVOIRS AND CRACK-INFLUENCE PARAMETERS}

To illustrate the Sayers and Kachanov (1991) crack-influence parameter method, consider the situation in which all the cracks in the system have the same vertical (or $z$-)axis of symmetry. (We use $x_{1}, x_{2}, x_{3}$ and $x, y, z$ notation interchangeably for the axes.) We assume the uncracked system is isotropic and, therefore, has the compliance matrix

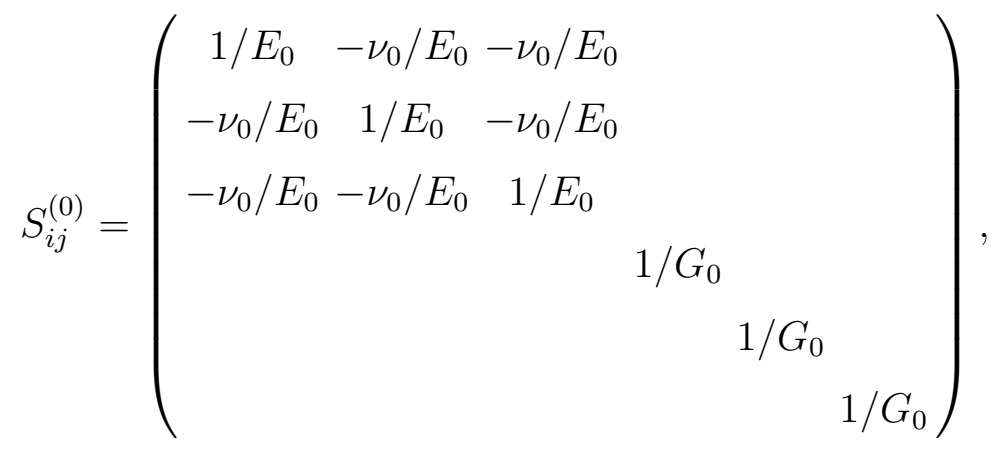


where $i, j=1,2,3$. In Eq. (9), $E_{0}$ is the background (assumed) isotropic Young's modulus, $G_{0}$ is the isotropic shear modulus, and $\nu_{0}$ is Poisson's ratio. Then, since the cracked/fractured system is not isotropic, we also have the first-order (meaning there is only one power of crack density $\rho_{c}$ present in our analysis) compliance correction matrix for horizontal fractures, which is:

$$
\Delta S_{i j}^{(1)}=\rho_{c}\left(\begin{array}{cccccc}
0 & 0 & \eta_{1} & & & \\
0 & 0 & \eta_{1} & & \\
\eta_{1} & \eta_{1} & 2\left(\eta_{1}+\eta_{2}\right) & & \\
& & & 2 \eta_{2} & \\
& & & & & \\
& & & & & \\
& & & & & 0
\end{array}\right) .
$$

The common factor $\rho_{c}$ in (10) is a scalar measure of the crack/fracture density. If the cracks are all penny-shaped having penny-radius $a$, and (presumed small) aspect ratio $\alpha=b / a$, then the volume of each penny crack is $v_{c}=4 \pi a^{2} b / 3=4 \pi \alpha a^{3} / 3$. The total crack density scalar is then often defined (Bristow, 1961) in these terms as $\rho_{c}=a^{3} N_{c}$, where $N_{c}$ is total number of such cracks in a unit volume of the reservoir material. If the cracks are all pennyshaped but perhaps have different crack radii $a_{i}$, then $\rho_{c}=\sum_{i=1, N} a_{i}^{3} / V \equiv N_{c}\left\langle a^{3}\right\rangle$, where $V$ is the total volume containing these $N_{c}$ cracks. For other crack shapes, the concept of $\rho_{c}$ has also been generalized (Budiansky and O'Connell, 1976).

Now it is not difficult to see that, if the cracks were oriented instead so that all their normals were pointed horizontally along the $x$-axis, then we would have one permutation of the matrix $\Delta S_{i j}^{(1)}$ and, if instead they were all pointed horizontally along the $y$-axis, then we would have another permutation of the matrix. If we wanted to obtain an isotropic compliance correction matrix, we could simply average these three permutations, i.e., just add these three $\Delta S^{\prime}$ 's together and then divide by three. [Note that this method of averaging, although correct for contributions linear in $\rho_{c}$, does not necessarily work for higher order corrections (Berryman, 2007).] This construction shows in part both the power and the simplicity of the Kachanov (1980) and Sayers and Kachanov (1991) approach — at least for these lower crack densities that now under consideration. The connection to the isotropic problem just noted is of great practical value, because it permits us to estimate the parameters $\eta_{1}$ and $\eta_{2}$ by studying isotropic cracked/fractured systems, and therefore permitting the use of well-understood effective medium theories, including the noninteraction (NI) ap- 
proximation (Zimmerman, 1991; Bažant and Planas, 1998; Berryman and Grechka, 2006). Formulas for $\eta_{1}$ and $\eta_{2}$ in the NI approximation are given in Table 1, together with examples of the values of $\eta_{1}, \ldots, \eta_{5}$ [from Berryman and Grechka (2006)] used later in our numerical studies. Parameters $\eta_{3}, \eta_{4}$, and $\eta_{5}$, are higher order (i.e., for higher powers of $\rho_{c}$ than linear) corrections that will be included in the computations, but not discussed in any detail in the present paper. For details, see Berryman and Grechka (2006) and Berryman (2007).

\section{ALIGNED HORIZONTAL FRACTURES AND VTI SYMMETRY}

Considering for now the simplest case of aligned horizontal fractures, as illustrated by the correction matrix (10), the axis of fracture symmetry is uniformly vertical, and so such a reservoir would exhibit VTI symmetry (assuming the background medium is isotropic). The resulting expressions for the Thomsen parameters in terms of the Sayers and Kachanov (1991) parameters $\eta_{1}$ and $\eta_{2}$ are found from a straightforward calculation to be

$$
\gamma_{h}=\frac{c_{66}-c_{44}}{2 c_{44}}=\rho_{c} \eta_{2} G_{0}
$$

and

$$
\epsilon_{h}=\frac{c_{11}-c_{33}}{2 c_{33}}=\rho_{c}\left[\left(1+\nu_{0}\right) \eta_{1}+\eta_{2}\right] \frac{E_{0}}{\left(1-\nu_{0}^{2}\right)} \simeq \frac{2 \rho_{c} \eta_{2} G_{0}}{1-\nu_{0}}
$$

The background shear modulus is $G_{0}$, and the corresponding Poisson ratio is $\nu_{0}$. Young's modulus is related to these two by $E_{0}=2\left(1+\nu_{0}\right) G_{0}$. We also find the important result that, for dry or drained cracks, $\delta_{h}=\epsilon_{h}$ to the lowest order in the crack density parameter. We have chosen to neglect the term in $\eta_{1}$ in the final expression of (12), as this is always less than $5 \%$ of the magnitude of $\eta_{2}$ and more typically on the order of a $1 \%$ correction to the term retained. (See TABLE 1.) Values of $\eta_{1}$ and $\eta_{2}$ can be determined from simulations and/or effective medium theories (Eshelby, 1957; Hill, 1963; Zimmerman, 1991; Berryman, 1995; Berryman and Grechka, 2006; Berryman, 2007). They depend on the elastic constants of the background medium, and on the shape of the cracks (assumed to be approximately penny-shaped in the present examples). Berryman (2007) also shows how to include higher order contributions in crack density to $\gamma_{h}, \epsilon_{h}$, and $\delta_{h}$, but we will not repeat these details here. 


\section{HTI RESERVOIR SYMMETRY, ALIGNED VERTICAL FRACTURES, AND WAVE SPEEDS IN THOMSEN'S APPROXIMATION}

One trick to carry our analytical results from the aligned horizontal fractures and VTI symmetry over to the aligned vertical fractures and HTI symmetry is this: First, we avoid making the effort to relabel the indices of the $c_{i j}$ 's. Instead we just change the meaning of the indices. [This amounts to using knowledge of Euler angles (Goldstein, 1950), and seismic wave reciprocity (Ben-Menahem and Singh, 1981) for these problems.] As long as we stay mentally oriented in the reference frame of the fractures themselves, we can continue to view the $x_{3}$-direction as the symmetry axis and the $x_{1} x_{2}$-plane as the plane of the fractures. The only change we need to make arises from the fact that the surface, where we shoot our seismic survey, is now at $90^{\circ}$ from the fracture plane, whereas for horizontal fractures the surface was parallel to and, therefore, at $0^{\circ}$ from the fracture plane. This observation implies that, wherever the angle $\theta$ (measured in radians) appeared in our previous formulas, now we must replace it by $\frac{\pi}{2}-\theta$ radians. So, $\sin ^{2} \theta \rightarrow \cos ^{2} \theta$ and vice versa in the various formulas. This algorithm is exactly right only for those planes that are both vertical and also perpendicular to the fracture plane, i.e., at azimuthal angles $\phi= \pm \frac{\pi}{2}$ measured from the fracture plane. Thus, to be correct for all angles, we actually need to replace $\sin ^{2} \theta$ by $\cos ^{2} \theta \sin ^{2} \phi$. And then, whenever $\phi=0$ or $\pi$, there is no angular dependence at all since this corresponds to the plane of the fracture - which is also the plane of isotropy.

For the remainder of this discussion, we limit ourselves to analysis of the behavior in the planes perpendicular to the vertical fractures, i.e, to the azimuthal angles $\phi= \pm \frac{\pi}{2}$. So it must be remembered that the following formulas apply only in these planes perpendicular to the plane of the aligned fractures/cracks. For other angles of propagation, we must also account for the azimuthal dependence on angle $\phi \neq \pm \frac{\pi}{2}$, but this is relatively easy to do.

For the $\theta$ dependence itself, taking $\sin ^{2} \theta \rightarrow 1-\sin ^{2} \theta$, is actually a handier way to proceed [see Rüger (2002) for an alternative approach], because we can then reduce all the formulas to a form equivalent to the one Thomsen originally used. It is also helpful to backup one step in Thomsen's derivation and restore squares, thereby unexpanding the square root. Certain approximations are then undone, and the final formulas we obtain will be more accurate.

If $\epsilon, \delta$, and $\gamma$ are the standard Thomsen parameters for the VTI symmetry (horizontal fracture), then the HTI results ( $\bar{v}$ is the HTI result for the speed with $\theta_{H}$ measured from 
the normal to the fracture plane) for $\mathrm{SH}$ waves corresponding to vertical fractures are:

$$
\bar{v}_{s h}^{2}\left(\theta_{H}\right)=v_{s}^{2}(0)\left[1+2 \gamma \cos ^{2} \theta_{H}\right]=v_{s}^{2}(0)(1+2 \gamma)\left[1-\frac{2 \gamma}{1+2 \gamma} \sin ^{2} \theta_{H}\right],
$$

where $\theta_{H}=\frac{\pi}{2}-\theta_{V}$ and $\theta_{V}=\theta$ is the angle measured from the normal to the fracture plane. From this result, we deduce that $\gamma \rightarrow \gamma_{H}=-\gamma /(1+2 \gamma)$, and $v_{s}^{2}(0) \rightarrow v_{s}^{2}(0)(1+2 \gamma)=v_{s h}^{2}\left(\frac{\pi}{2}\right)$, as might be expected. This is a rigorous statement for the form of the equation considered. Then, for weak anisotropy, the limit will be $\gamma \rightarrow-\gamma$; but this final approximation is neither necessary nor recommended for some of the higher crack densities considered here.

Calculations for $v_{s v}^{2}$ give the following HTI results:

$$
\bar{v}_{s v}^{2}\left(\theta_{H}\right)=v_{s}^{2}(0)\left[1+2\left[v_{p}^{2}(0)(1+2 \epsilon) / v_{s}^{2}(0)\right] \frac{\epsilon-\delta}{1+2 \epsilon} \sin ^{2} \theta_{H} \cos ^{2} \theta_{H}\right] .
$$

The calculation for $\bar{v}_{p}^{2}\left(\theta_{H}\right)$ is the most difficult one, so we will show some of the steps here. Starting with $v_{p}^{2}(\theta)=v_{p}^{2}(0)\left[1+2 \delta \sin ^{2} \theta \cos ^{2} \theta+2 \epsilon \sin ^{4} \theta\right]$, we make the substitution: $\theta=\frac{\pi}{2}-\theta_{H}$. Then,

$$
\begin{aligned}
\bar{v}_{p}^{2}\left(\theta_{H}\right) & =v_{p}^{2}(0)\left[1+2 \delta \sin ^{2} \theta_{H} \cos ^{2} \theta_{H}+2 \epsilon \cos ^{4} \theta_{H}\right] \\
& =v_{p}^{2}(0)\left[1+2 \epsilon+2[(\delta-2 \epsilon)+\epsilon] \sin ^{2} \theta_{H} \cos ^{2} \theta_{H}-2 \epsilon \sin ^{2} \theta_{H}\right] \\
& =v_{p}^{2}(0)(1+2 \epsilon)\left[1+\frac{2(\delta-2 \epsilon)}{1+2 \epsilon} \sin ^{2} \theta_{H} \cos ^{2} \theta_{H}-\frac{2 \epsilon}{1+2 \epsilon} \sin ^{4} \theta_{H}\right],
\end{aligned}
$$

which lead [when comparing to (5) and (6)] to the results $\epsilon \rightarrow \epsilon_{H}=\frac{-\epsilon}{1+2 \epsilon} \simeq-\epsilon$, and $\delta \rightarrow \delta_{H} \equiv \frac{\delta-2 \epsilon}{1+2 \epsilon} \simeq \delta-2 \epsilon$. As a consistency check, taking the difference of the results just quoted for $\epsilon_{H}$ and $\delta_{H}$ shows that $\epsilon_{H}-\delta_{H}=(\epsilon-\delta) /(1+2 \epsilon)$ holds for both (15) and (14), and in addition shows that $\epsilon_{H}-\delta_{H} \simeq(\epsilon-\delta)$ for weak anisotropy.

Similarly, the pertinent wave speeds are: $v_{p}(0) \rightarrow \bar{v}_{p}(0)=v_{p}(0) \sqrt{1+2 \epsilon}=$ $\sqrt{c_{33}(1+2 \epsilon) / \rho}=\sqrt{c_{11} / \rho}$, while $v_{s h}(0) \rightarrow \bar{v}_{s h}(0)=v_{s}(0) \sqrt{1+2 \gamma}=\sqrt{c_{44}(1+2 \gamma) / \rho}=$ $\sqrt{c_{66} / \rho}$ in (13)-(15). But the remaining velocity $\bar{v}_{s v}(0)$ does not change since $v_{s v}(\theta)$ is completely symmetric in $\theta$ for Thomsen's approximation, and therefore has to remain so (also having the same end points) after the switch from $\theta$ to $\frac{\pi}{2}-\theta$. Of course, $v_{s v}(0)=v_{s v}\left(\frac{\pi}{2}\right)$ as well, so $\bar{v}_{s v}(0)=v_{s v}(0)$ is also rigorously true. These results were (almost) all known previously and can also be found in Rüger (2002), p. 75, but the present derivation may seem more intuitive (i.e., a little less technical) for some readers. Note, however, that the present defintion of $\delta_{H} \equiv \frac{\delta-2 \epsilon}{1+2 \epsilon}$ differs from a related (but not conceptually identical) choice of definition made by Rüger (2002). 
The final results for HTI symmetry in Thomsen's approximation are now easily obtained from the preceding results:

$$
\begin{gathered}
\bar{v}_{s h}\left(\theta_{H}\right) \simeq v_{s}(0) \sqrt{1+2 \gamma}\left[1-\frac{\gamma}{1+2 \gamma} \sin ^{2} \theta_{H}\right], \\
\bar{v}_{s v}\left(\theta_{H}\right) \simeq v_{s}(0)\left[1+\left[v_{p}^{2}(0)(1+2 \epsilon) / v_{s}^{2}(0)\right] \frac{\epsilon-\delta}{1+2 \epsilon} \sin ^{2} \theta_{H} \cos ^{2} \theta_{H}\right],
\end{gathered}
$$

and

$$
\bar{v}_{p}\left(\theta_{H}\right) \simeq v_{p}(0) \sqrt{1+2 \epsilon}\left[1-\frac{\epsilon}{1+2 \epsilon} \sin ^{2} \theta_{H}+\frac{\delta-\epsilon}{1+2 \epsilon} \sin ^{2} \theta_{H} \cos ^{2} \theta_{H}\right],
$$

where (18) follows directly from the second expression in (15) [which was purposely left in a nonreduced state to make this transition easier]. To be completely internally consistent with the approximate formulas, the $2 \epsilon$ and $2 \gamma$ contributions to the denominators should be removed, but this step should make little difference in practice.

Examples of these results for small $\left(\rho_{c}=0.05\right)$ and higher $\left(\rho_{c}=0.1,0.2\right)$ crack densities are presented in Figure 1. See Berryman and Grechka (2006) for details of the methods used to obtain all the Sayers and Kachanov crack-influence parameters from computer simulation results and Berryman (2007) for a full discussion of the reservoir application.

Some features of the individual figures in Figure 1 are worthy of further discussion. In particular, it is very interesting to see that for the HTI system all of the SH curves in Fig. 1(b) start at the same value for $\theta_{H}=0$, and similarly for Fig. 1(e). The analytical reason for this behavior follows easily from equations (9) and (10). Since there is no correction to $S_{66}$ from (10), and furthermore, no coupling to any of the other coefficients, we have immediately that $c_{66}=1 / S_{66}=G_{0}$. So this result is universal for systems of aligned vertical cracks, and is therefore both expected and observed in the two SH-wave figures.

Although something similar seems to be happening for the P-wave speeds in Fig. 1(a), the same is not true for Fig. 1(d). The reason that Fig. 1(d) does not follow this pattern is seen from equations (9) and (10) again. Whereas the shear modes in the lower right corner of the matrix in Eq. (9) are completely uncoupled from the rest of the matrix, and the same is true of corrections in Eq. (10), this is not true for the $3 \times 3$ submatrices in the upper left corners. In general, these terms are coupled (and especially so for the $c_{i j}$ 's, since these coefficients are found by inversion of the matrices $S+\Delta S$ shown - so an inverse of the pertinent $3 \times 3$ determinant contains all these coupling terms). There is, however, one (and only one) case where this situation simplifies, and that is when Poisson's ratio of the host medium $\nu_{0}=0.0$. 
Then, the upper left $3 \times 3$ of Eq. (9) is diagonal. Furthermore, since $\left|\eta_{1}\right|<<\eta_{2}$, the upper left $3 \times 3$ of Eq. (10) is very nearly diagonal as well. Thus, it is expected that $c_{11}$ is constant (or nearly so) for all values of $\rho_{c}$, and therefore all the $v_{p}$ curves converge at $\theta_{H}=0$ in this case - as is observed here in Fig. 1(a). However, this does not happen, and should not happen, for Fig. 1(d) since $\nu_{0} \neq 0.0$ in this example, and so the cross-coupling becomes significant.

An alternative physical argument (thanks to S. Nakagawa, private communication) for the effects observed in (a), (b), and (e) comes from the fact that velocities do not change because wave particle motion does not alter the crack shape (i.e., no stress is applied to the crack). The difference between (a) and (d) comes because non-zero Poisson's ratios result in an opening/closing motion of the cracks (while, for $\nu_{0}=0$, cracks never change shape). However, for the cases (c) and (f), results always depend on crack density because shear stress is always present on the cracks.

\section{EXTENDING THOMSEN'S FORMULATION TO LARGER OFFSETS FOR VTI AND HTI SYMMETRY}

The most obvious problem with Thomsen's approximations to the wave speeds generally occurs in $v_{s v}(\theta)$. As noted previously, the key issue here is that Thomsen's approximation for $v_{s v}(\theta)$ is completely symmetric around $\theta=\pi / 4=45^{\circ}$, while - unfortunately - this is usually not true of the actual wave speeds $v_{s v}(\theta)$. This inherent error may seem innocuous in itself since it is not immediately clear whether it affects the results for small angles of incidence $\left(<15^{\circ}\right)$ or not, but this inaccuracy clearly does lead to large over- or underestimates of wave speeds in the neighborhood of both the extreme value (i.e., a peak or a trough) located at $\theta=\theta_{e x}$ and also in the neighborhood of $\theta=45^{\circ} \neq \theta_{e x}$. So these discrepancies can certainly become issues at offsets larger than the original design criterion of $15^{\circ}$.

To improve this situation while still making use of a simple and practical approximation to the phase speed, we reconsider an approach originally proposed in Berryman (1979). In particular, notice that the square root formula for $R(\theta)$ in Eq. (3) can be exactly and conveniently rewritten as:

$$
R(\theta)=\left[c_{11} \sin ^{2} \theta+c_{33} \cos ^{2} \theta-c_{44}\right] \sqrt{1-\zeta(\theta)}
$$


where

$$
\zeta(\theta) \equiv \frac{\left[\left(c_{11}-c_{44}\right)\left(c_{33}-c_{44}\right)-\left(c_{13}+c_{44}\right)^{2}\right] \sin ^{2} 2 \theta}{\left[c_{11} \sin ^{2} \theta+c_{33} \cos ^{2} \theta-c_{44}\right]^{2}} .
$$

To simplify this expression, first notice that $\zeta(\theta)$ has an absolute maximum (or minimum) value, which occurs when $\theta$ takes the value $\theta_{m}$ determined by

$$
\tan ^{2} \theta_{m}=\frac{c_{33}-c_{44}}{c_{11}-c_{44}} \equiv 1-x_{m},
$$

where

$$
x_{m}=\frac{c_{11}-c_{33}}{c_{11}-c_{44}}>0 .
$$

The inequality in (22) is true for VTI media having horizontal fractures, since for this case the stiffness difference $c_{11}-c_{33}$ has already been shown in (12) to be positive (as it is also known that $\rho_{c}, \eta_{2}$, and $G_{0}$ are all positive, and Poisson's ratio satisfies $\left.\nu_{0} \leq \frac{1}{2}\right)$.

Then, the extreme value $\zeta_{m} \equiv \zeta\left(\theta_{m}\right)$ is given by

$$
\zeta_{m}=1-\frac{\left(c_{13}+c_{44}\right)^{2}}{\left(c_{11}-c_{44}\right)\left(c_{33}-c_{44}\right)}=\frac{2(\epsilon-\delta) c_{33}}{c_{11}-c_{44}}=\frac{2(\epsilon-\delta) v_{p}^{2}(0)}{v_{p}^{2}(0)(1+2 \epsilon)-v_{s}^{2}(0)},
$$

where the second and third expressions relate $\zeta_{m}$ to the difference between the Thomsen parameters $\epsilon$ and $\delta$, and also to $v_{p}(0)$ and $v_{s}(0)$. In general, $(\epsilon-\delta)$ - and therefore $\zeta_{m}$ take values positive, negative, or zero. Furthermore, $\zeta(\theta)$ can be rewritten as

$$
\zeta(\theta)=\frac{2 \zeta_{m}}{1+\chi(\theta)}
$$

where

$$
\chi(\theta)=\frac{1}{2}\left[\frac{\tan ^{2} \theta}{\tan ^{2} \theta_{m}}+\frac{\tan ^{2} \theta_{m}}{\tan ^{2} \theta}\right] .
$$

It is always true that $\zeta(\theta) \leq 1$. [Note that $\zeta_{m} \geq 0$ for all layered media since $\epsilon-\delta \geq 0$ for layered elastic media (Postma, 1955; Backus, 1962; Berryman, 1979). However, such a simple constraint is not known for other types of anisotropic systems.] The square root in equation (19), can be expanded to first order as

$$
\sqrt{1-\zeta(\theta)} \simeq 1-\frac{\zeta(\theta)}{2}=1-\frac{\zeta_{m}}{1+\chi(\theta)} .
$$

Approximate results for $v_{p}(\theta)$ and $v_{s v}(\theta)$ are therefore:

$$
v_{p}^{2}(\theta) \simeq \frac{1}{\rho}\left\{\left[c_{11} \sin ^{2} \theta+c_{33} \cos ^{2} \theta\right]-\frac{\zeta_{m}\left[\left(c_{11}-c_{44}\right) \sin ^{2} \theta+\left(c_{33}-c_{44}\right) \cos ^{2} \theta\right]}{2[1+\chi(\theta)]}\right\}
$$


and

$$
v_{s v}^{2}(\theta) \simeq \frac{1}{\rho}\left\{c_{44}+\frac{\zeta_{m}\left[\left(c_{11}-c_{44}\right) \sin ^{2} \theta+\left(c_{33}-c_{44}\right) \cos ^{2} \theta\right]}{2[1+\chi(\theta)]}\right\} .
$$

The only approximation made in arriving at equations (27) and (28) was the approximation of the square root shown in (26).

Although this simple approach is the one most commonly used, the analysis is not really limited to using only the first order Taylor approximation in (26). Other researchers (Fowler, 2003; Pederson et al., 2007) have explored rational approximations to such square roots, but we choose to take a rather different approach.

Progress is made by noting that the quantity $\frac{1}{2}[1+\chi(\theta)]$ may be rewritten as:

$$
\frac{1}{2}[1+\chi(\theta)]=\frac{1}{4}\left(\frac{\tan \theta}{\tan \theta_{m}}+\frac{\tan \theta_{m}}{\tan \theta}\right)^{2}=\frac{\left(\tan ^{2} \theta+\tan ^{2} \theta_{m}\right)^{2}}{4 \tan ^{2} \theta \tan ^{2} \theta_{m}} .
$$

To simplify this expression, first multiply numerator and denominator of (29) by $\cos ^{4} \theta \cos ^{4} \theta_{m}$. The denominator of the result is then proportional to $\sin ^{2} 2 \theta \sin ^{2} 2 \theta_{m}$, while the numerator is now proportional to the square of the quantity

$$
\cos ^{2} \theta \cos ^{2} \theta_{m}\left(\tan ^{2} \theta+\tan ^{2} \theta_{m}\right)=\sin ^{2} \theta \cos ^{2} \theta_{m}+\sin ^{2} \theta_{m} \cos ^{2} \theta=\frac{1}{2}\left(1-\cos 2 \theta \cos 2 \theta_{m}\right) .
$$

Combining equations (29) and (30) gives

$$
\zeta(\theta)=\frac{\zeta_{m} \sin ^{2} 2 \theta_{m} \sin ^{2} 2 \theta}{\left[1-\cos 2 \theta_{m} \cos 2 \theta\right]^{2}}
$$

which (although this may not be immediately obvious) is just a more compact version of (20). Equation (31) is exact; no approximations were made in the transition from (20) to (31).

Note, from (30), we also have (by dividing through by $2 \cos ^{2} \theta_{m}$ ) that

$$
\sin ^{2} \theta+\tan ^{2} \theta_{m} \cos ^{2} \theta=\frac{\left[1-\cos 2 \theta_{m} \cos 2 \theta\right]}{2 \cos ^{2} \theta_{m}} .
$$

The exact expression (2) for quasi-SV-wave speed can now be rewritten as

$$
2 \rho v_{s v}^{2}=2 c_{44}+\left(c_{11}-c_{44}\right)\left(\sin ^{2} \theta+\tan ^{2} \theta_{m} \cos ^{2} \theta\right)[1-\sqrt{1-\zeta(\theta)}] .
$$

Similarly, the exact equation for quasi-P-wave speed becomes

$$
2 \rho v_{p}^{2}=2 c_{44}+\left(c_{11}-c_{44}\right)\left(\sin ^{2} \theta+\tan ^{2} \theta_{m} \cos ^{2} \theta\right)[1+\sqrt{1-\zeta(\theta)}] .
$$


Both of these expressions are exact rearrangements of the original equations.

These results can be consolidated further by using either the identities $\sin ^{2} \theta_{1} \cos ^{2} \theta_{2}+$ $\sin ^{2} \theta_{2} \cos ^{2} \theta_{1}=\sin ^{2}\left(\theta_{1}+\theta_{2}\right)+\sin ^{2}\left(\theta_{2}-\theta_{1}\right)$, or the result (32), together with the definition

$$
c_{11}+c_{33}-2 c_{44}=\frac{c_{11}-c_{44}}{\cos ^{2} \theta_{m}} \equiv 2 \Delta c .
$$

So finally, we have two compact results for the exact wave speeds:

$$
\rho v_{s v}^{2}=c_{44}+\frac{\Delta c}{2}\left[1-\cos 2 \theta_{m} \cos 2 \theta\right][1-\sqrt{1-\zeta(\theta)}]
$$

for the quasi-SV-wave speed, and also the corresponding equation which is

$$
\rho v_{p}^{2}=c_{44}+\frac{\Delta c}{2}\left[1-\cos 2 \theta_{m} \cos 2 \theta\right][1+\sqrt{1-\zeta(\theta)}]
$$

for the exact quasi-P-wave speed.

\section{Quasi-SV-wave extreme point $\theta_{e x}$}

One test of the usefulness of these equations is to check how close the value $\theta_{m}$ is to the value of the angle $\theta_{e x}$ at which the extreme value (either peak or trough) of the quasiSV wave speed occurs. We will present two distinct calculations of this extreme point: one follows immediately, while another more technical method is summarized in Appendix B.

Start from (36), and take the first term in the Taylor series expansion of $\sqrt{1-\zeta(\theta)}$. Then, the quantity that needs to be maximized or minimized is proportional to:

$$
g(\theta)=\frac{\sin ^{2} 2 \theta}{1-\cos 2 \theta_{m} \cos 2 \theta} .
$$

The extremum occurs (by definition) at $\theta=\theta_{e x}$ when the first derivative vanishes, so this implies

$$
\frac{d g(\theta)}{d \theta}=\frac{2 \sin 2 \theta}{\left[1-\cos 2 \theta_{m} \cos 2 \theta\right]^{2}}\left\{2\left(\cos 2 \theta-\cos 2 \theta_{m}\right)+\sin ^{2} 2 \theta \cos 2 \theta_{m}\right\}=0 .
$$

The solutions of (39) for $\sin 2 \theta=0$ are the ones pertinent for the end points, not for the extreme point in the middle range of $\theta$ that we are after. Note also that $\theta_{e x}=45^{\circ}$ is not a solution of the equation, not even when $\theta_{m}=45^{\circ}$. The curly bracketed expression gives a quadratic formula for $\cos 2 \theta$, and the physical solution of this formula (an unphysical second "solution" implies a cosine value greater than unity) is

$$
\cos 2 \theta=\frac{1-\sin 2 \theta_{m}}{\cos 2 \theta_{m}} .
$$


Using well-known trigonometric identities, this formula can be transformed into

$$
1-2 \sin ^{2} \theta=\frac{1-2 \sin \theta_{m} \cos \theta_{m}}{1-2 \sin ^{2} \theta_{m}} .
$$

This intermediate result can then be shown to be equivalent to both

$$
\sin ^{2} \theta_{e x}=\frac{\tan \theta_{m}}{1+\tan \theta_{m}} \quad \text { and } \quad \cos ^{2} \theta_{e x}=\frac{1}{1+\tan \theta_{m}} .
$$

Taking the ratio of these two forms gives the useful final (though approximate) result:

$$
\tan ^{2} \theta_{e x}=\tan \theta_{m}
$$

This formula is not exact because we made use of approximation (26) to simplify the equations. (Both a precise definition and more precise formula for $\theta_{e x}$ are presented in Appendix B.)

We can better understand the meaning of (43) in the following way: It is important to know whether the (true) peak of the quasi-SV-wave is actually at $\theta=45^{\circ}$, or $\theta=\theta_{m}$, or somewhere else. Since $\tan 45^{\circ}=1$, and $\tan 0^{\circ}=0$, we see that $0<\tan \theta_{m}<1$ for VTI anisotropic media. Furthermore, from (21) we have $\tan \theta_{m} \equiv\left(\tan 45^{\circ}-x_{m}\right)^{1 / 2} \simeq 1-\frac{x_{m}}{2}$, where $x_{m}$ was defined in (22) and may legitimately be assumed to be a relatively small quantity in the present context. Then,

$$
\tan \theta_{e x}=\left(1-x_{m}\right)^{1 / 4} \simeq 1-\frac{x_{m}}{4}=1-\frac{c_{11}-c_{33}}{4\left(c_{11}-c_{44}\right)} .
$$

From this point of view, we see that the extreme point location $\theta_{e x}$ is intermediate between $\theta_{m}$ and $45^{\circ}$. In terms of this tangent measure of the angle $\theta_{e x}$ at the extreme point, it is exactly the geometric mean of the two quantities: $\tan 45^{\circ}=1$ and $\tan \theta_{m} \simeq 1-x_{m} / 2$.

The estimate of $\theta_{e x}$ determined by (43) is called $\theta_{e x}^{(1)}$ in the examples of TABLE 1. A second, and somewhat more accurate, estimate of $\theta_{e x}^{(2)}$ is obtained in Appendix B, and also illustrated in TABLE 1. Examples of the results for $\theta_{m}, \theta_{e x}^{(1)}$, and $\theta_{e x}^{(2)}$, based on the same fracture models discussed previously, are displayed and compared to the actual (both exact and approximate) $v_{s v}$ curves in Figure 2. Further discussion will be delayed until the end of this section, so we can present and then discuss some further developments.

\section{Extended Thomsen formulas for VTI symmetry}

A direct comparison with Thomsen's approximations uses equations (36) and (37) to arrive at approximate formulas for $v_{s v}(\theta)$ and $v_{p}(\theta)$ analogous to Thomsen's. The resulting 
expressions are

$$
v_{p}^{2}(\theta) / v_{p}^{2}(0) \simeq 1+2 \epsilon \sin ^{2} \theta-\frac{\epsilon-\delta}{2} \frac{2 \sin ^{2} \theta_{m} \sin ^{2} 2 \theta}{\left[1-\cos 2 \theta_{m} \cos 2 \theta\right]},
$$

from which we find:

$$
v_{p}(\theta) / v_{p}(0) \approx 1+\epsilon \sin ^{2} \theta-\frac{\epsilon-\delta}{4} \frac{2 \sin ^{2} \theta_{m} \sin ^{2} 2 \theta}{\left[1-\cos 2 \theta_{m} \cos 2 \theta\right]}
$$

Similarly,

$$
v_{s v}^{2}(\theta) / v_{s}^{2}(0) \simeq 1+\left[v_{p}^{2}(0) / v_{s}^{2}(0)\right] \frac{\epsilon-\delta}{2} \frac{2 \sin ^{2} \theta_{m} \sin ^{2} 2 \theta}{\left[1-\cos 2 \theta_{m} \cos 2 \theta\right]},
$$

from which follows:

$$
v_{s v}(\theta) / v_{s}(0) \approx 1+\left[v_{p}^{2}(0) / v_{s}^{2}(0)\right] \frac{\epsilon-\delta}{4} \frac{2 \sin ^{2} \theta_{m} \sin ^{2} 2 \theta}{\left[1-\cos 2 \theta_{m} \cos 2 \theta\right]} .
$$

Equations (45) and (47) are approximate results obtained by expanding one square root in a Taylor series, while (46) and (48) are further approximations obtained by expanding another square root in a Taylor series. Note that these expressions reduce precisely to (5)-(7) if $\theta_{m} \equiv 45^{\circ}$.

Now the factor $\sin ^{2} 2 \theta /\left[1-\cos 2 \theta_{m} \cos 2 \theta\right]$ contains the terms $\sin ^{2} 2 \theta=4 \sin ^{2} \theta \cos ^{2} \theta-$ obviously completely symmetric under the interchange of $\sin ^{2} \theta$ and $\cos ^{2} \theta$ - and this is important for translation to the HTI case where $\theta_{H}=\frac{\pi}{2}-\theta$. So this term simply does not change in the VTI to HTI transition. Then note that the factor $\cos 2 \theta=\cos ^{2} \theta-\sin ^{2} \theta$ is antisymmetric under the interchange of $\sin ^{2} \theta$ and $\cos ^{2} \theta$, so $\cos 2 \theta \rightarrow-\cos 2 \theta_{H}$ when switching from the VTI to the HTI points of view.

Next, comparing pairs of equations (46) and (48) to (5) and (6), the differences are found to lie in a factor of the form:

$$
\frac{2 \sin ^{2} \theta_{m}}{\left[1-\cos 2 \theta_{m} \cos 2 \theta\right]} \rightarrow \frac{1}{2 \cos ^{2} \theta_{m}} \quad \text { as } \quad \theta \rightarrow \theta_{m}
$$

which depends explicitly on the angle $\theta_{m}$ determined by $\tan ^{2} \theta_{m}=\left(c_{33}-c_{44}\right) /\left(c_{11}-c_{44}\right)$, and — for $\theta \neq \theta_{m}$ - also on $\theta$ itself. As indicated, the expression goes to $1 / 2 \cos ^{2} \theta_{m}$ in the limit of $\theta \rightarrow \theta_{m}$. But, since $\sin ^{2} \theta_{m}=\tan ^{2} \theta_{m} /\left(1+\tan ^{2} \theta_{m}\right)$ and $\cos 2 \theta_{m}=\left(1-\tan ^{2} \theta_{m}\right) /\left(1+\tan ^{2} \theta_{m}\right)$, other useful identities are

$$
\sin ^{2} \theta_{m}=\frac{c_{33}-c_{44}}{c_{11}+c_{33}-2 c_{44}}=1-\cos ^{2} \theta_{m}
$$


and

$$
\cos 2 \theta_{m}=\frac{c_{11}-c_{33}}{c_{11}+c_{33}-2 c_{44}}=1-2 \sin ^{2} \theta_{m} .
$$

Then, to make the formulas (46) and (48) look as much as possible like Thomsen's formulas - and thereby arrive at a somewhat different understanding of the equations (5) and (6) for "weak anisotropy," first eliminate $\theta_{m}$ by arbitrarily setting it equal to $\theta_{m}=45^{\circ}$, in

which case $\sin ^{2} \theta_{m}=\frac{1}{2}$ and $\cos 2 \theta_{m}=0$. We see that Thomsen's formulas (5) and (6) are recovered exactly. So we conclude that Thomsen's formulas are based not only on an explicit assumption of weak anisotropy, but also on an implicit assumption that $\theta_{m}$ is so close to $45^{\circ}$ that these differences may be safely negelected. The examples already presented in Figure 1 show that this implicit assumption is valid for low crack densities below 0.05 , but not valid for higher crack densities such as 0.1 and above.

\section{Extended Thomsen formulas for HTI symmetry}

To complete this analysis, we show now the results for HTI symmetry that arise when the fractures/cracks are aligned and vertical. These results follow easily from the discussion immediately following equations (45)-(48):

$$
\bar{v}_{p}^{2}\left(\theta_{H}\right) / v_{p}^{2}(0)(1+2 \epsilon) \simeq 1-\frac{2 \epsilon}{1+2 \epsilon} \sin ^{2} \theta_{H}-\frac{\epsilon-\delta}{2(1+2 \epsilon)} \frac{2 \sin ^{2} \theta_{m} \sin ^{2} 2 \theta_{H}}{\left[1+\cos 2 \theta_{m} \cos 2 \theta_{H}\right]},
$$

from which we find:

$$
\bar{v}_{p}\left(\theta_{H}\right) / v_{p}(0) \sqrt{1+2 \epsilon} \approx 1-\frac{\epsilon}{1+2 \epsilon} \sin ^{2} \theta_{H}-\frac{\epsilon-\delta}{4(1+2 \epsilon)} \frac{2 \sin ^{2} \theta_{m} \sin ^{2} 2 \theta_{H}}{\left[1+\cos 2 \theta_{m} \cos 2 \theta_{H}\right]} .
$$

Similarly,

$$
\bar{v}_{s v}^{2}\left(\theta_{H}\right) / v_{s}^{2}(0) \simeq 1+\left[v_{p}^{2}(0) / v_{s}^{2}(0)\right] \frac{\epsilon-\delta}{2} \frac{2 \sin ^{2} \theta_{m} \sin ^{2} 2 \theta_{H}}{\left[1+\cos 2 \theta_{m} \cos 2 \theta_{H}\right]},
$$

from which follows:

$$
\bar{v}_{s v}\left(\theta_{H}\right) / v_{s}(0) \approx 1+\left[v_{p}^{2}(0) / v_{s}^{2}(0)\right] \frac{\epsilon-\delta}{4} \frac{2 \sin ^{2} \theta_{m} \sin ^{2} 2 \theta_{H}}{\left[1+\cos 2 \theta_{m} \cos 2 \theta_{H}\right]} .
$$

Again, these formulas reduce exactly to the equivalent Thomsen formulas for HTI symmetry given previously in (16)-(18) if $\theta_{m} \rightarrow 45^{\circ}$. These results also have exactly the same significance as those for VTI symmetry in (45)-(48); however, it should always be remembered that these formulas apply only in planes perpendicular to the plane of the aligned fractures/cracks. For other angles of propagation, we must also account for the azimuthal dependence on angle $\phi$. 


\section{The two critical angles}

For both the VTI and HTI cases there are two critical angles, $\theta_{m}$ [extreme point of $\zeta(\theta)$ ] and $\theta_{e x}$ [extreme point of the quasi-SV-wave speed]. Technical Appendices A and B develop methods of estimating these two critical angles for the VTI symmetry from Thomsen's parameters and, therefore, from seismic data. TABLE 1 shows the types of results expected for a range of real anisotropic materials and earth media (note that presence or absence of cracks was not considered during the selection of these examples). Results show how $\theta_{m}$ and $\theta_{e x}$ differ from each other, and how successful the methods developed in the Appendix B are for the computation of $\theta_{e x}$.

\section{DISCUSSION AND CONCLUSIONS}

The analysis presented here of the phase velocity equations does not depend on the source of the anisotropy, and therefore can be applied to layered media, etc., as well as to fractured media as we have done here.

The Kachanov (1980) and Sayers and Kachanov (1991) crack-influence parameters are ideally suited to analyzing the role of fracture mechanics in producing anisotropic elastic constants for aligned fractures in a reservoir exhibiting VTI or HTI symmetry. When this approach is combined with poroelastic analysis through the use of Skempton's (1954) coefficient (as was done here in Appendix C), it becomes very easy to analyze a wide range of complicated situations that may raise in reservoir analysis, such as trying to deduce whether the fractures are dry/drained, or fluid-saturated/undrained. Skempton's coefficient $B$ introduces a single parameter that varies from 0 to 1 as fluid properties change from being negligible to being very strong influences on the fracture compliance - and therefore on the Thomsen seismic parameters..

Another important observation from the modeling presented is that the Thomsen weak anisotropy formulation is valid for crack densities up to about $\rho_{c} \simeq 0.05$, but should be replaced by more accurate approximations, or (better yet) exact calculations whenever possible when the crack density is much above 0.05 . If the crack density is $\rho_{c} \simeq 0.1$, or higher, then higher accuracy approximations are essential. Conversely, if the crack density $\rho_{c}$ estimated from seismic data using the weak anisotropy formulation is in fact larger than $\rho_{c} \simeq 0.05$, 
one conclusion we might reach is that a more accurate method is required both to verify and properly quantify the result.

\section{ACKNOWLEDGMENTS}

The author thanks V. Grechka, M. Kachanov, S. Nakagawa, S. R. Pride, M. Schoenberg, and I. Tsvankin for helpful collaborations and conversations. Work performed under the auspices of the U.S. Department of Energy by the University of California, Lawrence Berkeley National Laboratory under Contract No. DE-AC03-76SF00098 and supported specifically by the Geosciences Research Program of the DOE Office of Basic Energy Sciences, Division of Chemical Sciences, Geosciences and Biosciences.

\section{REFERENCES}

Backus G. E., 1962. Long-wave elastic anisotropy produced by horizontal layering. Journal of Geophysical Research 67, 4427-4440.

Bakulin A., Grechka V., and Tsvankin, I., 2000. Estimation of fracture parameters from reflection seismic data - Part I: HTI model due to a single fracture set: Geophysics $\mathbf{6 5}$ (6), 1788-1802.

Bažant Z. P. and Planas J., 1998. Fracture and Size Effect in Concrete and Other Quasibrittle Materials, CRC Press, Boca Raton, Florida, pp. 560-563.

Ben-Menahem A. and Singh S. J., 1981. Seismic Waves and Sources, Dover, Mineola, New York, pp. 156, 163, 173.

Berryman J. G., 1979. Long-wave elastic anisotropy in transversely isotropic media. Geophysics 44, 896-917.

Berryman J. G., 1995. Mixture theories for rock properties. In American Geophysical Union Handbook of Physical Constants, edited by T. J. Ahrens, AGU, New York, pp. 205-228.

Berryman J. G., 1999. Origin of Gassmann's equations. Geophysics 64, 1627-1629. 
Berryman J. G., 2007. Seismic waves in rocks with fluids and fractures. Geophysical Journal International 171, 954-974.

Berryman J. G., 2008. Exact seismic velocities for transversely isotropic media and extended Thomsen formulas for stronger anisotropies. Geophysics 73, D1-D10.

Berryman J. G. and Grechka V., 2006. Random polycrystals of grains containing cracks: Model of quasistatic elastic behavior for fractured systems. Journal of Applied Physics $100,113527$.

Biot M. A. and Willis D. G., 1957. The elastic coefficients of the theory of consolidation. J. Appl. Mech. 24, 594-601.

Bristow J., 1960. Microcracks and the static and dynamic elastic constants of annealed and heavily cold-worked metals. British J. Appl. Phys. 11, 81-85.

Brown R. J. S. and Korringa J., 1975. On the dependence of the elastic properties of a porous rock on the compressiblity of a pore fluid. Geophysics 40, 608-616.

Budiansky B. and O'Connell R. J., 1976. Elastic moduli of a cracked solid. International Journal of Solids and Structures 12, 81-97.

Eshelby J. D., 1957. The determination of the elastic field of an ellipsoidal inclusion, and related problems. Proc. Roy. Soc. London A 241, 376-396.

Fowler P., 2003. Practical VTI approximations: A systematic anatomy. Journal of Applied Geophysics 54, 347-367.

Gassmann F., 1951. Über die elastizität poroser medien. Vierteljahrsschrift der Naturfoschenden Gesellschaft in Zürich 96, 1-23.

Goldstein, H., 1950. Classical Mechanics, Addison-Wesley, Reading, Massachusetts, pp. 107108.

Hill, R., 1963. Elastic properties of reinforced solids: Some theoretical principles. J. Mech. Phys. Solids 11, 357-372.

Hornby B. E., 1994. The Elastic Properties of Shales, Ph. D. Thesis, Cambridge, UK. 
Hsu C.-J. and Schoenberg M., 1993. Elastic waves through a simulated fractured medium. Geophysics 58, 964-977.

Kachanov M., 1980. Continuum model of medium with cracks. ASCE J. Engineering Mech. 106, 1039-1051.

Landau L. D. and Lifshitz E. M., 1986. Theory of Elasticity, Butterworth-Heinemann, Oxford, pp. 124-127.

Lyakhovitsky F. M., 1993. Velocity of $P$-waves in transversely isotropic media. Canadian Journal of Exploration Geophysics 29, 114-116.

Lynn H. B., Simon K. M., Layman M., and Schneider R., 1995. Use of anisotropy in P-wave and $S$-wave data for fracture characterization in a naturally fractured gas reservoir. The Leading Edge 14, 887-893.

Musgrave M. J. P., 2003. Crystal Acoustics: Acoustical Society of America, Leetsdale, Pennsylvania, Chapter 8 .

Pederson Ø., Ursin B., and Stovas A., 2007. Wide-angle phase-slowness approximations. Geophysics 72, S177-S185.

Postma G. W., 1955. Wave propagation in a stratified medium. Geophysics 20, 780-806.

Rüger A., 2002. Reflection Coefficients and Azimuthal AVO Analysis in Anisotropic Media, Geophysical Monographs Series, Number 10, SEG, Tulsa, OK.

Sayers C. M. and Kachanov M., 1991. A simple technique for finding effective elastic constants of cracked solids for arbitrary crack orientation statistics. International Journal of Solids and Structures 27, 671-680.

Schoenberg, M., 1980. Elastic wave behavior across linear slip interfaces. J. Acoust. Soc. Am. 68, 1516-1521.

Skempton A. W., 1954. The pore-pressure coefficients $A$ and B. Géotechnique 4, 143-147.

Thomsen L., 1986. Weak elastic anisotropy. Geophysics 51, 1954-1966. 
Thomsen L., 2002. Understanding Seismic Anisotropy in Exploration and Exploitation, 2002 Distinguished Instructor Short Course, Number 5, SEG, Tulsa, OK.

Tsvankin I., 1997. Anistoropic parameter and $P$-wave velocity for orthorhombic media. Geophysics 62, 1292-1309.

Tsvankin I., 2005. Seismic Signatures and Analysis of Reflection Data in Anisotropic Media, handbook of Geophysical Exploration, Seismic Exploration, Volume 29, Elsevier, Amsterdam.

Vernik L. and Liu X., 1997. Velocity anisotropy in shales: A petrophysical study. Geophysics 62, 521-532.

Zimmerman R. W., 1991. Compressibility of Sandstones, Elsevier, Amsterdam.

\section{APPENDIX A: COMPUTING $\theta_{m}$ FROM SEISMIC DATA}

The angle $\theta_{m}$ at $\zeta(\theta)$ takes it maximum value is easily found using [see (21)]

$$
\tan ^{2} \theta_{m}=\frac{c_{33}-c_{44}}{c_{11}-c_{44}}=\frac{v_{p}^{2}(0)-v_{s}^{2}(0)}{\left(c_{11} / \rho\right)-v_{s}^{2}(0)}
$$

and

$$
\epsilon=\frac{c_{11}-c_{33}}{2 c_{33}}=\frac{c_{11} / \rho-v_{p}^{2}(0)}{2 v_{p}^{2}(0)},
$$

showing that

$$
\tan ^{2} \theta_{m}=1-x_{m}=\frac{v_{p}^{2}(0)-v_{s}^{2}(0)}{(1+2 \epsilon) v_{p}^{2}(0)-v_{s}^{2}(0)}
$$

So we also have

$$
x_{m}=\frac{2 \epsilon v_{p}^{2}(0)}{(1+2 \epsilon) v_{p}^{2}(0)-v_{s}^{2}(0)} .
$$

Thus, $\theta_{m}$ is determined using the same data as in the standard analysis of seismic reflection data, which also determines the various small angle wave speeds and the three main Thomsen parameters.

The pertinent fixed factors for use in the formulas (50) and (51) are given by

$$
\sin ^{2} \theta_{m}=\frac{v_{p}^{2}(0)-v_{s}^{2}(0)}{2\left[(1+\epsilon) v_{p}^{2}(0)-v_{s}^{2}(0)\right]}
$$


and

$$
\cos 2 \theta_{m}=\frac{\epsilon v_{p}^{2}(0)}{(1+\epsilon) v_{p}^{2}(0)-v_{s}^{2}(0)}=1-2 \sin ^{2} \theta_{m} .
$$

And, finally, equations (23), (31), and (58) also fix the extreme value of $\zeta_{m}=\zeta\left(\theta_{m}\right)$, as well as its angular location $\theta_{m}$ (as already shown), using the same data.

\section{APPENDIX B: EXTREME POINT $\theta_{e x}$ FOR QUASI-SV-WAVE SPEED}

The result (43) in the main text was obtained by first making the assumption that $\zeta_{m}-$ and therefore the magnitude of $\zeta(\theta)$ - was so small that it did not significantly influence the location of the angle $\theta_{e x}$ for the extreme value of the quasi-SV-wave speed. We can improve on this result, but the analysis is much more involved than what was shown previously.

After some tedious algebra, it can be shown that the exact formula for the extreme point is given by the formula

$$
\cos 2 \theta_{e x}=\cos 2 \theta_{m}\left[1-\left(1-\cos ^{2} \theta_{e x}\right) F\left(\zeta_{e x}\right)\right]
$$

where

$$
F(\zeta)=\frac{1-\sqrt{1-\zeta}}{\zeta} \sqrt{1-\zeta}
$$

and with $\zeta=\zeta(\theta)$ as defined previously in (31). Note that the formula (62) reduces correctly [see equation (39)] to $(40)$ when $\left|\zeta_{m}\right|<<1$, since $F(\zeta) \rightarrow \frac{1}{2}$ in this limit.

Additional tedious algebra and use of some trigonometic identities shows that, if $\zeta_{m}$ is small but not negligible, then (43) is replaced by

$$
\tan ^{2} \theta_{e x} \simeq \tan \theta_{m}+\frac{\zeta_{m}}{4 \sin \theta_{m}}\left(1-\tan \theta_{m}\right)^{2}\left(1+\tan \theta_{m}\right)
$$

Equation (64) shows that the sign of $\zeta_{m}$ matters to some degree, since $\zeta_{m}>0$ will push the value of $\theta_{e x}$ closer to $45^{\circ}$, while $\zeta_{m}<0$ will push the value closer to $\theta_{m}$. The estimate of $\theta_{e x}$ determined by $(64)$ is labelled $\theta_{e x}^{(2)}$ in the examples of TABLE 2.

Of the examples computed for presentation in TABLE 2, the only material that proved difficult was zinc. For this one anisotropic material, the estimate (64) proved to be invalid due to the failure of the assumption that $\zeta_{m}(=1.957$ for zinc) was small compared to unity. However, it was nevertheless possible to solve (62) directly by iteration, starting with the value of $\theta_{m}$ substituted for $\theta_{e x}$ on the right hand side of the equation and then solving for 
$\theta_{e x}^{i}$ at each step $i$ on the left. This process converged to five significant figures after ten iterations. When the same iterative procedure was applied to apatite, the result converged after only four iterations to $42.05^{\circ}$ [differing only slightly from that of the formula (64)]. For Mesaverde sandstone, the iterative procedure converges to $41.72^{\circ}$ in two iterations. The author does not however recommend the use of (43) in this iterative mode as it may give the user a false sense of exactness of the result, and no analysis has been done yet to determine whether or not there are potentially multiple solutions (all but one of which must be spurious if they exist) of the equation. So the iterative procedure should be used (if at all) with some caution.

Examples of the use of these formulas are also discussed in the main text and illustrated in Figure 2.

\section{APPENDIX C: FLUID EFFECTS IN ANISOTROPIC FRACTURED MEDIA}

Berryman (2007) treats the effects of fluids in anisotropic fractured media, but limited the discussion to VTI symmetry. In the present work, Eqs. (16)-(18) and also Eqs. (52)-(55) show the formulas needed to interpret the Thomsen parameters correctly for HTI media. These two strands of the theory will be combined here to show how fluids in fractures affect the HTI coefficients.

The pertinent formulas for the VTI case of horizontal fractures are Eqs. (46), (47), and (68) from Berryman (2007), which are:

$$
\begin{gathered}
\gamma_{V T I}=\rho_{c} G_{0} D_{2}, \\
\epsilon_{V T I}=\frac{2 \rho_{c} G_{0}}{1-\nu_{0}} D_{1},
\end{gathered}
$$

and

$$
\delta_{V T I}=\frac{2 \rho_{c} G_{0}}{1-\nu_{0}}\left[\frac{2\left(1-\nu_{0}\right) D_{1}-\left(1-2 \nu_{0}\right) D_{2}}{1+2\left(1-\nu_{0}\right) \rho_{c} G_{0}\left(D_{2}-D_{1}\right)}\right]
$$

where

$$
D_{1} \equiv \eta_{2}+\rho_{c}\left(\eta_{3}+\eta_{5}\right) \simeq \eta_{2}
$$

and

$$
D_{2} \equiv \eta_{2}+\rho_{c} \eta_{5} \simeq \eta_{2}
$$

Although $D_{1}$ and $D_{2}$ appear to be equivalent (or nearly so for the low crack density limit) in these formulas, their roles in the theory are quite distinct, as $D_{1}$ contributes to compliances 
involving principal stresses and linear strains, while $D_{2}$ contributes only to compliances directly involving shear stresses and strains. This means that the two are affected differently by the presence of fluid in the fractures. Berryman (2007) shows explicitly that the fracture compliances involving only shear stresses and strains are not affected by the presence of fluid in the fractures so $D_{2} \rightarrow D_{2}$, when a fluid is added.

In contrast, the fracture compliances involving the principal stresses and linear strains must be multiplied by a factor $(1-B)$ in the presence of fluids, where $B$ is Skempton's (1954) coefficient. Thus, addition of fluid to the fractures implies $D_{1} \rightarrow D_{1}(1-B)$. Normally $B$ itself is a function of the fluid bulk modulus $K_{f}$, the bulk modulus of the surrounding isotropic medium $K_{0}$, the porosity $\phi$, the drained bulk modulus of the porous system $K_{d}$, and the Biot-Willis coefficient $\alpha=1-K_{d} / K_{0}$, according to:

$$
1-B=\frac{1-K_{f} / K_{0}}{1-K_{f} / K_{0}+\left(K_{f} \alpha / \phi K_{d}\right)} .
$$

See Gassmann (1951), Skempton (1954), Biot and Willis (1957), Brown and Korringa (1975), and Berryman (1999) for background. For present purposes, the main point is that $B$ typically lies in the range $0 \leq B \leq 1$, where $B=0$ corresponds to fractures containing air but no liquids, while $B=1$ corresponds to a fluid saturant with a very large bulk modulus comparable to that of the surrounding matrix material (i.e., $K_{f} \simeq K_{0}$ ).

Using these results to compute the effective values of the Thomsen parameters for the VTI case, we have

$$
\begin{gathered}
\gamma_{V T I} \simeq \rho_{c} \eta_{2} G_{0}, \\
\epsilon_{V T I} \simeq \frac{2 \rho_{c} \eta_{2} G_{0}(1-B)}{1-\nu_{0}},
\end{gathered}
$$

and

$$
\delta_{V T I} \simeq \frac{2 \rho_{c} \eta_{2} G_{0}}{1-\nu_{0}}\left[\frac{2\left(1-\nu_{0}\right)(1-B)-\left(1-2 \nu_{0}\right)}{1+2\left(1-\nu_{0}\right) \rho_{c} \eta_{2} G_{0} B}\right]=\frac{2 \rho_{c} \eta_{2} G_{0}}{1-\nu_{0}}\left[\frac{1-2\left(1-\nu_{0}\right) B}{1+2\left(1-\nu_{0}\right) \rho_{c} \eta_{2} G_{o} B}\right] .
$$

We do not need to consider $\gamma$ further, since it is independent of fluid properties. Equations (71)-(73) are general for ellipsoidal cracks, and for Biot-Gassmann consistency (Gassmann, 1950; Biot and Willis, 1957) of the predicted saturating-fluid effects. From general theorems in the elasticity of heterogeneous media (Hill, 1963), the for of these results also apply - at least approximately - to any crack-shapes that can be reasonably fitted by some ellipsoidal 
shape; then, the values of the factors $\eta_{1}$ and $\eta_{2}$ need to be recomputed for the closest fitting ellipsoid.

For VTI, we have two distinct limiting cases: $B=0$ and $B=1$ (actual values of $B$ will normally be intermediate to these two). For the dry/drained case, we have $B=0$, so:

$$
\epsilon_{V T I}=\frac{2 \rho_{c} \eta_{2} G_{0}}{1-\nu_{0}}=\delta_{V T I}>0 .
$$

The result is simply that $\epsilon_{V T I}=\delta_{V T I}$ for an air-filled, horizontal fracture system. In contrast, when $B=1$, we have

$$
\epsilon_{V T I} \equiv 0,
$$

and

$$
\delta_{V T I} \simeq-\frac{2 \rho_{c} \eta_{2}\left[1-2\left(1-\nu_{0}\right)\right] G_{0}}{\left(1-\nu_{0}\right)\left[1+2\left(1-\nu_{0}\right) \rho_{c} \eta_{2} G_{o}\right]}<0 .
$$

We can make further use of these results for the HTI system, since those results are expressible in terms of the VTI formulas. (In the following equations, unsubscripted expressions for $\delta$ and $\epsilon$ are those for VTI just given above.) As discussed following Eq. (15), the general results valid for all $B$ are:

$$
\delta \rightarrow \delta_{H T I}=\frac{\delta-2 \epsilon}{1+2 \epsilon},
$$

and

$$
\epsilon-\delta \rightarrow \epsilon_{H T I}-\delta_{H T I}=\frac{\delta-\epsilon}{1+2 \epsilon} .
$$

For the case, $B=0$ we then have:

$$
\delta_{H T I}=\frac{-\delta}{1+2 \delta},=\epsilon_{H T I} .
$$

For $B=1$, we have:

$$
\delta_{H T I}=\delta<0,
$$

since $\epsilon=0$ for VTI and $B=1$; and, for $B=1$, we have:

$$
\epsilon_{H T I}=\delta_{H T I}+\frac{\epsilon-\delta}{1+2 \epsilon}=\delta_{H T I}-\delta=0 .
$$

So for fluid-filled cracks having $B=1$, we find that $\epsilon_{V T I}=\epsilon_{H T I}=0$.

All these results are consistent with those of Bakulin et al. (2000) [see their Fig. 6], although their analysis was based in part on penny-shaped cracks (as we have done here) and also in part on the linear slip model of fractures (Schoenberg, 1980). 
TABLE 1. Formulas for the first two (linear) fracture influence-parameters from the non-interaction (NI) approximation, and values of five fracture-influence parameters for the two models considered by Berryman and Grechka (2006). Note that, since Poisson's ratio $\nu_{0}$ of the background medium is bounded above by $\nu_{0} \leq \frac{1}{2}$, the first two crack influence parameters satisfy $\left|\eta_{1}\right| \leq \eta_{2} / 18$.

\begin{tabular}{|c|c|c|c|}
\hline \hline Fracture-influence & NI & First Model & Second Model \\
Parameters & Approx. & $\nu_{0}=0.00$ & $\nu_{0}=0.4375$ \\
\hline$\eta_{1}\left(\mathrm{GPa}^{-1}\right)$ & $-\frac{\nu_{0} \eta_{2}}{2\left(5-\nu_{0}\right)}$ & 0.0000 & -0.0192 \\
$\eta_{2}\left(\mathrm{GPa}^{-1}\right)$ & $\frac{8\left(1-\nu_{0}\right)\left(5-\nu_{0}\right)}{15\left(2-\nu_{0}\right) G_{0}}$ & 0.1941 & 0.3994 \\
$\eta_{3}\left(\mathrm{GPa}^{-1}\right)$ & - & -0.3666 & -1.3750 \\
$\eta_{4}\left(\mathrm{GPa}^{-1}\right)$ & - & 0.0000 & 0.0000 \\
$\eta_{5}\left(\mathrm{GPa}^{-1}\right)$ & - & 0.0917 & 0.5500 \\
\hline \hline
\end{tabular}


TABLE 2. Examples of $\zeta_{m}$ - i.e., the extreme value $\zeta\left(\theta_{m}\right)$ - and its angular location $\theta_{m}$ for various rocks and minerals. The angular location of the extreme point of $v_{s v}(\theta)$ is also given approximately by the two estimates $\theta_{e x}^{(1)}$ and $\theta_{e x}^{(2)}$ defined in the text. The data for $\epsilon$, $\delta, v_{p}(0)$, and $v_{s}(0)$ for the first six examples are all taken from Table 1 of Thomsen (1986), the next five examples are from Table 1 of Lyakhovitsky (1993), and the final five examples are from Appendix A of Vernik and Liu (1997).

\begin{tabular}{|c|c|c|c|c|c|c|c|c|}
\hline Sample & $\epsilon$ & $\delta$ & $v_{p}(0)(\mathrm{m} / \mathrm{s})$ & $v_{s}(0)(\mathrm{m} / \mathrm{s})$ & $\zeta_{m}$ & $\theta_{m}$ & $\theta_{e x}^{(1)}$ & $\theta_{e x}^{(2)}$ \\
\hline Cotton Valley shale & 0.135 & 0.205 & 4721. & 2890. & -0.1564 & $39.89^{\circ}$ & $42.43^{\circ}$ & $42.38^{\circ}$ \\
\hline Mesaverde sandstone & 0.081 & 0.057 & 3688. & 2774 . & 0.0805 & $40.48^{\circ}$ & $42.73^{\circ}$ & $42.75^{\circ}$ \\
\hline Muscovite crystal & 1.12 & -0.235 & 4420. & 2091. & 0.8985 & $26.90^{\circ}$ & $35.46^{\circ}$ & $39.69^{\circ}$ \\
\hline Pierre shale & 0.015 & 0.060 & 2202. & 969. & -0.1076 & $44.48^{\circ}$ & $44.74^{\circ}$ & $44.74^{\circ}$ \\
\hline Taylor sandstone & 0.110 & -0.035 & 3368. & 1829. & 0.3135 & $41.12^{\circ}$ & $43.06^{\circ}$ & $43.12^{\circ}$ \\
\hline Wills Point shale & 0.215 & 0.315 & 1058. & 387. & -0.1543 & $39.27^{\circ}$ & $42.12^{\circ}$ & $42.05^{\circ}$ \\
\hline Apatite & 0.096 & 0.586 & 6.605 & 4.552 & -1.367 & $40.55^{\circ}$ & $42.77^{\circ}$ & $42.43^{\circ}$ \\
\hline $\mathrm{BaTiO}_{3}$ & -0.055 & -0.046 & 5.669 & 3.047 & -0.030 & $47.40^{\circ}$ & $46.20^{\circ}$ & $46.20^{\circ}$ \\
\hline Beryl & 0.061 & -0.168 & 9.462 & 4.885 & 0.535 & $42.80^{\circ}$ & $43.90^{\circ}$ & $43.93^{\circ}$ \\
\hline$\beta$-quartz & 0.028 & -0.048 & 6.454 & 3.689 & 0.208 & $43.86^{\circ}$ & $44.43^{\circ}$ & $44.43^{\circ}$ \\
\hline Zinc & 0.780 & 2.655 & 2.947 & 2.365 & -1.957 & $23.32^{\circ}$ & $33.29^{\circ}$ & $33.56^{\circ}$ \\
\hline \multicolumn{9}{|l|}{ Monterey shale } \\
\hline$\phi=0.050$ & 0.11 & 0.19 & 4.54 & 2.77 & -0.190 & $40.67^{\circ}$ & $42.83^{\circ}$ & $42.79^{\circ}$ \\
\hline$\phi=0.166$ & 0.20 & -0.06 & 3.62 & 2.27 & 0.517 & $37.82^{\circ}$ & $41.38^{\circ}$ & $41.72^{\circ}$ \\
\hline$\phi=0.296$ & 0.22 & 0.05 & 2.68 & 1.79 & 0.342 & $36.74^{\circ}$ & $40.83^{\circ}$ & $41.13^{\circ}$ \\
\hline \multicolumn{9}{|l|}{ North Sea shale } \\
\hline$\phi=0.012$ & 0.24 & 0.02 & 3.86 & 2.22 & 0.383 & $37.35^{\circ}$ & $41.14^{\circ}$ & $41.43^{\circ}$ \\
\hline$\phi=0.029$ & 0.29 & 0.19 & 3.20 & 2.00 & 0.168 & $35.59^{\circ}$ & $40.23^{\circ}$ & $40.43^{\circ}$ \\
\hline
\end{tabular}



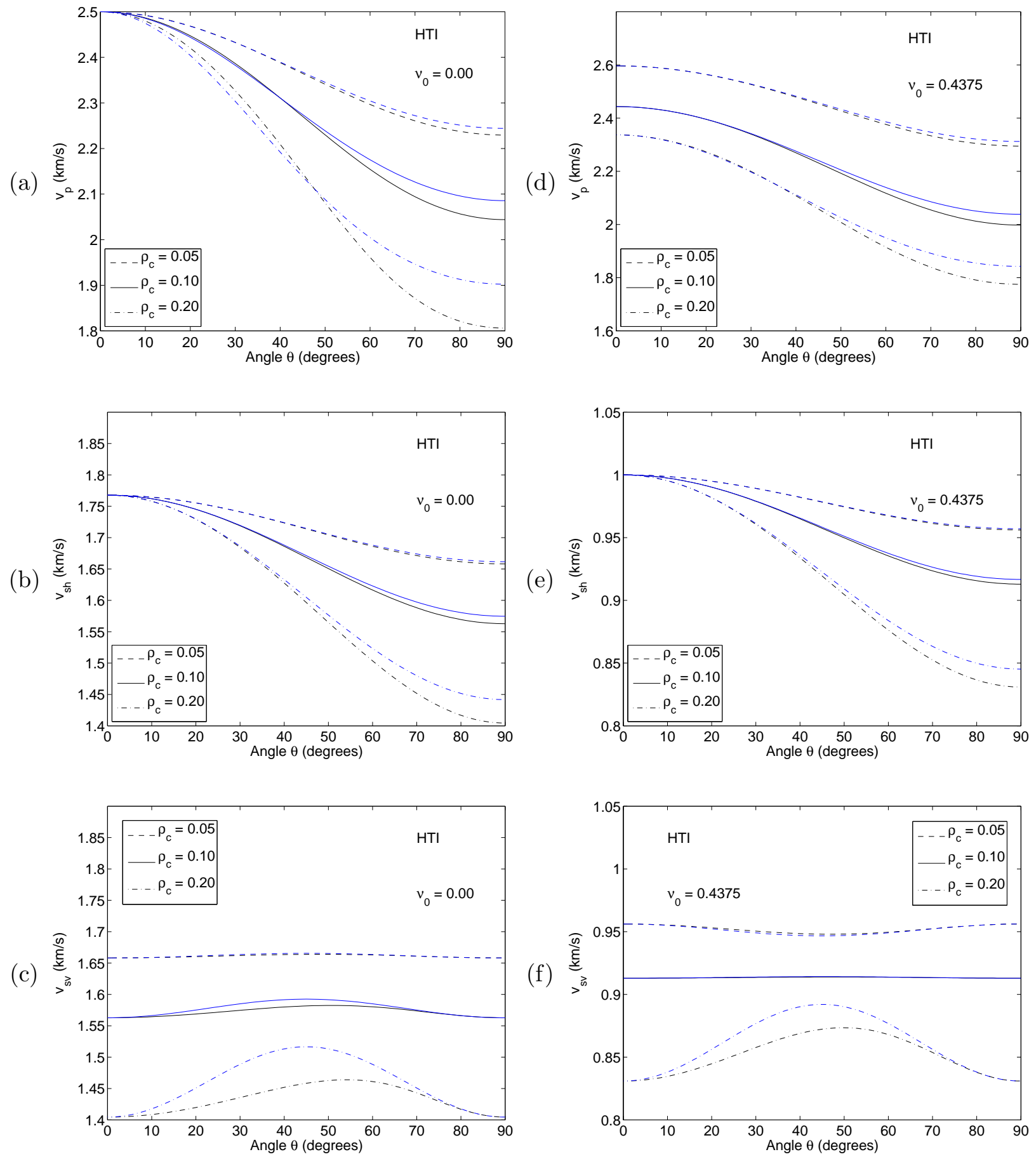

FIG. 1: For vertical cracks: examples of anisotropic quasi- $P$ compressional wave speed $\left(v_{p}\right), S H$ shear wave speed $\left(v_{s h}\right)$, and quasi- $S V$ shear wave speed $\left(v_{s v}\right)$ for two values of Poisson's ratio $\nu_{0}$ of the host medium: (a)-(c) $\nu_{0}=0.00,(\mathrm{~d})-(\mathrm{f}) \nu_{0}=0.4375$. Velocity curves in black are exact for the fracture model discussed in the text. The Thomsen weak anisotropy velocity curves for the same fracture model are then overlain in blue. Significant discrepancies observed between the exact values and Thomsen's approximation for $v_{s v}$ in both cases provide a primary motivation for the present work. 

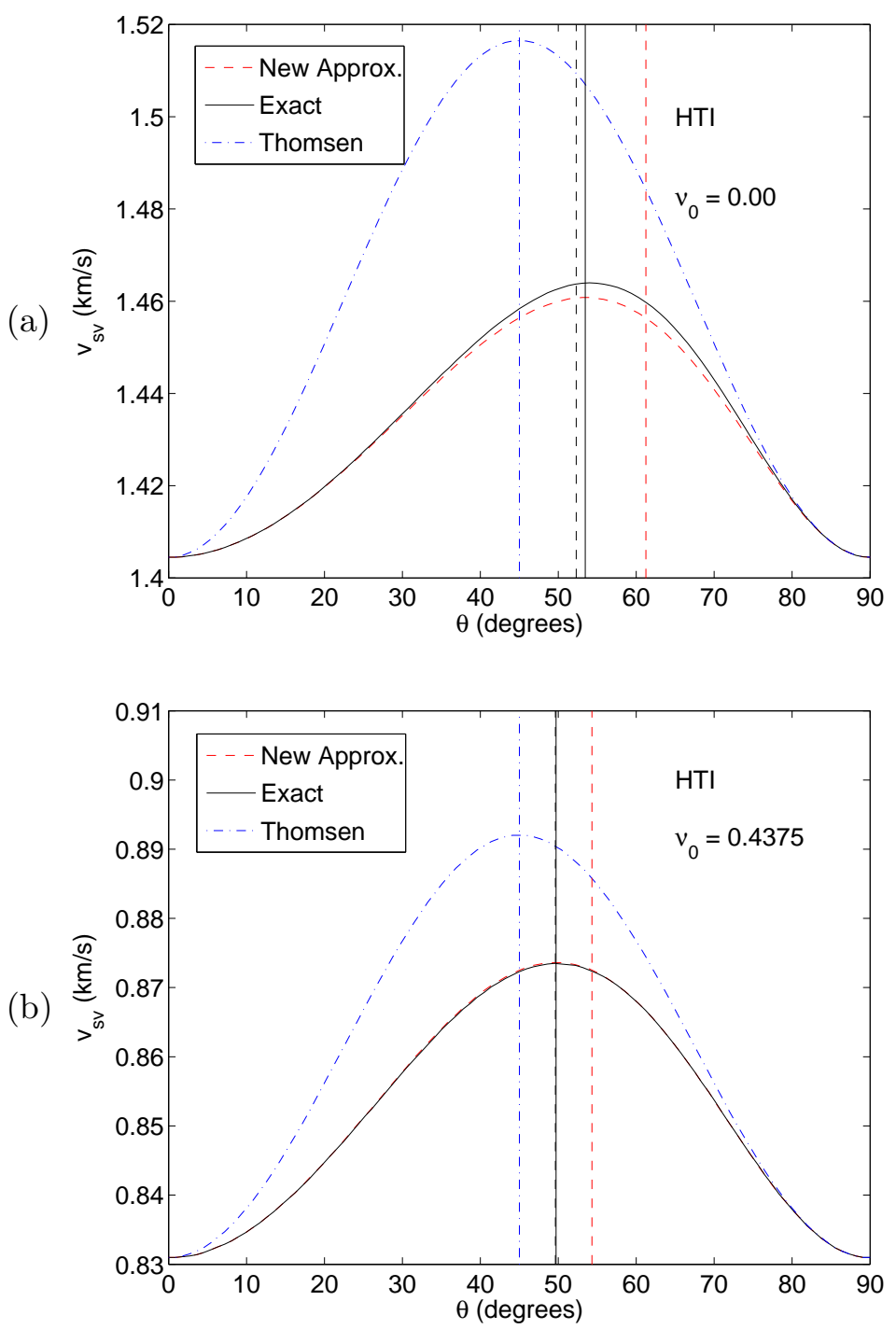

FIG. 2: For aligned vertical cracks having crack density $\rho_{c}=0.2$ and HTI overall symmetry: examples of anisotropic quasi- $S V$ shear wave speed $\left(v_{s v}\right)$ for two values of Poisson's ratio $\nu_{0}$ of the host medium: (a)-(b) $\nu_{0}=0.00$, (c) - (d) $\nu_{0}=0.4375$. Velocity curves in red are those for the new approximation presented in the text The exact result for the fracture/crack model discussed in the text is then overlain in black. Finally, the Thomsen weak anisotropy velocity curves for the same fracture/crack model are overlain in blue. To show how the approximations for the angular location $\theta_{e x}$ compare to the actual peaks, vertical lines are plotted to indicate these locations. The value $\theta_{e x}=45^{\circ}$ is always exact for Thomsen's approximation. The value $\theta_{m}$ as determined by (21) is shown in red. The values of $\theta_{e x}^{(1)}$ from (43) are shown as a black dashed line, while $\theta_{e x}^{(2)}$ from (62) are shown as a black solid line. The two estimates $\theta_{e x}^{(1)}$ and $\theta_{e x}^{(2)}$ are nearly indistinguishable (at this level of resolution) for for $\nu_{0}=0.4375$, as are the exact and new approximate values of the $v_{s v}$ wave speeds. 

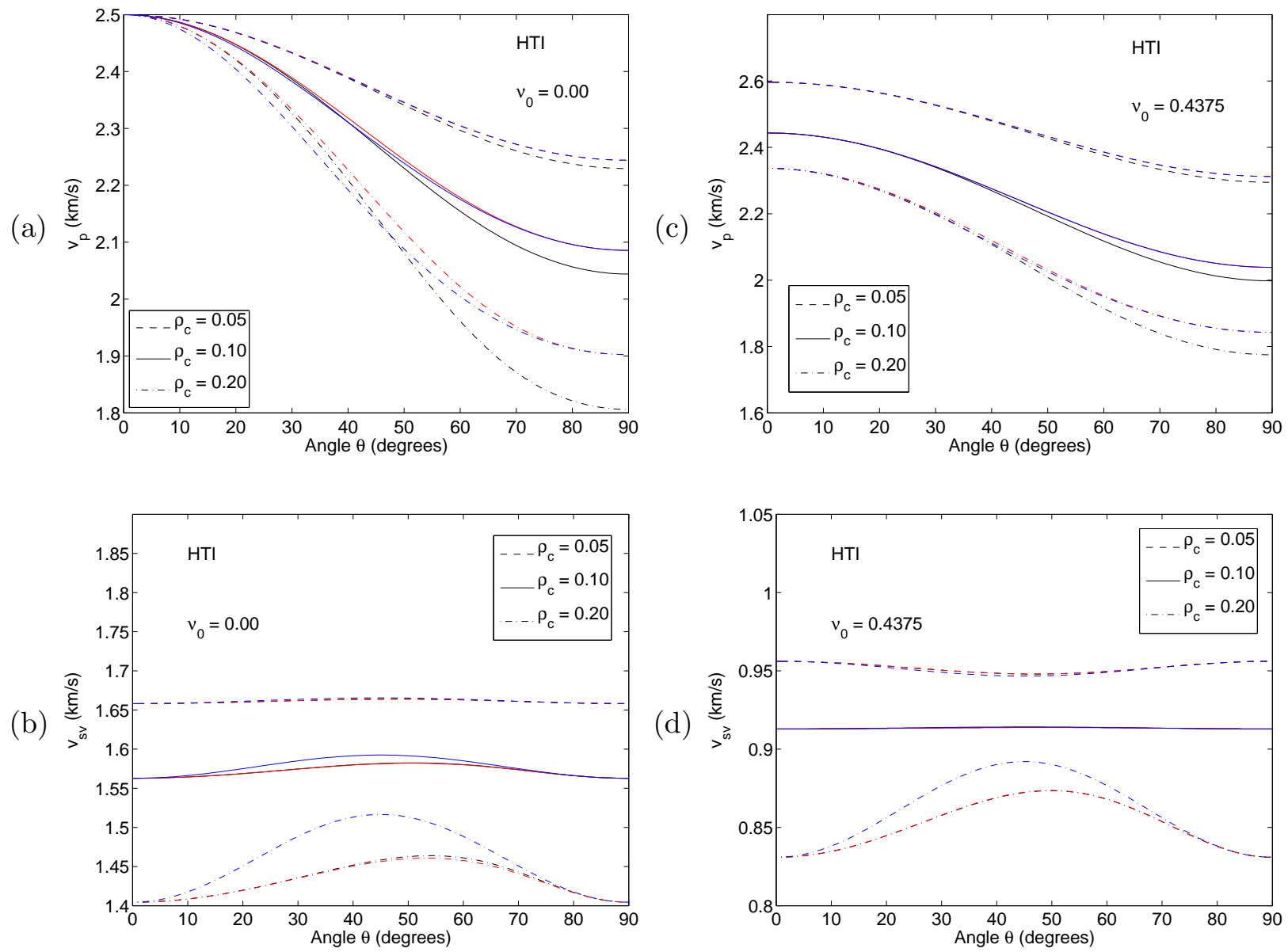

FIG. 3: For aligned vertical cracks, and HTI symmetry: examples of anisotropic quasi- $P$ compressional wave speed $\left(v_{p}\right)$ and quasi- $S V$ shear wave speed $\left(v_{s v}\right)$ for two values of Poisson's ratio $\nu_{0}$ of the host medium: (a)-(b) $\nu_{0}=0.00$, (c) - (d) $\nu_{0}=0.4375$. Velocity curves in black are exact for the fracture/crack model discussed in the text. The new approximation presented in the text is then overlain in red. Finally, the Thomsen weak anisotropy velocity curves for the same fracture/crack model are overlain in blue. Plots for $v_{s h}(\theta)$ are not affected by the new approximation scheme, and therefore are not displayed here. 